\title{
Patologia aguda nos cuidados de saúde primários: um estudo transversal descritivo da realidade numa USF
}

Ana Aires, ${ }^{1}$ André Ferreira, ${ }^{1}$ Alcino Santos, ${ }^{1}$ Rafael Gonçalves ${ }^{1}$

\section{RESUMO}

Objetivo: Caracterizar a consulta de agudos (CA) realizada numa Unidade de Saúde Familiar (USF) ao longo de quatro anos, de acordo com a frequência dos motivos de consulta/diagnóstico e destino dos utentes.

Tipo de estudo: Estudo transversal, descritivo e analítico, aplicado à clínica.

Local: USF Alpha, ACeS Baixo Vouga.

População: Utentes que recorreram à CA de uma USF de janeiro-2014 a dezembro-2017.

Métodos: Foram estudados todos os episódios de CA ao longo de quatro anos $(n=25.810)$, com recolha de dados relativamente a sexo, idade, distribuição temporal da CA; frequência da CA por utente; diagnóstico e destino dos utentes. Foi feita uma análise descritiva e inferencial com testes não-paramétricos utilizando o programa informático Statistical Package for the Social Sciences (SPSS ${ }^{\circledR}$ ), v. 25.0.

Resultados: Dos episódios de CA, a maioria eram mulheres $(n=15.596,60,4 \%, p<0,001)$, com mediana de idade de 40 anos e uma frequência/pessoa de dois episódios no período descrito. A maioria das CA teve lugar no primeiro trimestre do ano, em janeiro, à segunda-feira e às primeiras horas do dia. As patologias mais prevalentes em contexto de CA foram a infeção aguda do aparelho respiratório superior $(n=3.744,17,8 \%)$, amigdalite aguda $(n=1.326,6,3 \%)$, cistite/infeção urinária $(n=987,4,7 \%)$, gastroenterite $(n=846,4,0 \%)$ e bronquite/bronquiolite aguda $(n=737,3,5 \%)$. Há diferenças significativas no encaminhamento entre médico de família do utente e atendimento por outro médico, encaminhando este último mais para o serviço de urgência $(p<0,001)$ e não havendo diferenças no que diz respeito à necessidade de chamar o INEM $(p=0,417)$.

Conclusão: Constatou-se haver diferenças de cuidados de saúde entre médicos de família, criando-se a necessidade de haver algoritmos de decisão e de estratificação de gravidade como estratégia de melhoria da acessibilidade e qualidade de cuidados prestados. A educação para a saúde dos utentes é uma estratégia fundamental a trabalhar em contexto de patologia aguda.

Palavras-chave: Cuidados de saúde primários; Patologia aguda; Consulta aberta; Portugal.

\section{INTRODUÇÃO}

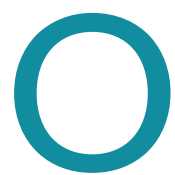

s cuidados de saúde primários (CSP) são o pilar central do sistema de saúde e a sua organização assenta no trabalho constante de melhoria dos cuidados prestados, com vista a uma melhor acessibilidade, proximidade e qualidade, com subsequente aumento da satisfação

1. Médico. USF Alpha, ACeS Baixo Vouga, Ovar. dos utentes. ${ }^{1}$ De acordo com a definição europeia de medicina geral e familiar (MGF) de 2002, espera-se ainda que os CSP proporcionem um acesso aberto e ilimitado aos seus utentes, garantindo a gestão de problemas, quer agudos quer crónicos, e mantendo o valor da equidade e do acesso aos CSP. ${ }^{2}$ Tais pressupostos exigem uma excelente capacidade organizativa das Unidades de Saúde Familiar (USF) de modo a dar resposta a todos os utentes, quer num sistema de consultas no próprio dia quer sob a forma de consultas programadas a curto ou médio prazo. 
Deste modo, a resposta à patologia aguda ou a agudizações de patologia crónica está prevista em contexto de consultas no próprio dia. A estas consultas é dada a designação genérica de consulta de agudos (CA) ou consulta do próprio dia e pode ser realizada pelo próprio médico de família ou por outro médico da unidade em sistema de intersubstituição (SIS), quando esgotada a capacidade assistencial do próprio dia pelo médico de família ou na sua ausência. ${ }^{3}$

Em Portugal, e mesmo a nível internacional, o tema da CA ao nível dos CSP encontra-se pouco estudado. As USF, atendendo ao seu modo organizativo que prevê uma autonomia organizativa, funcional e técnica, não dispõem de linhas orientadoras homogéneas que definam o modo de funcionamento da CA. ${ }^{4}$ Para além disso, verifica-se uma escassez de estudos de investigação que caracterizem a CA. Dois estudos nacionais de 2002 tentam caracterizar a utilização dos serviços de atendimento ao nível dos CSP através de consultas urgentes $^{5-6}$ e um estudo mais recente, de 2018, tenta também avaliar o perfil do utilizador da consulta de agudos, bem como os principais motivos para a mesma. ${ }^{7}$ Em ambos os estudos constatou-se que o género feminino era o principal utilizador da CA, sendo a patologia infeciosa, em particular a patologia do foro respiratório, o principal motivo de recorrência à CA. .-7 $^{5-1}$

O objetivo global do presente estudo foi avaliar e caracterizar a CA. Para esse efeito caracterizou-se a CA realizada numa USF ao longo de quatro anos (janeiro de 2014 a dezembro de 2017), de acordo com a frequência dos motivos de consulta/diagnóstico e destino dos utentes.

\section{MÉTODOS}

Tratou-se de um estudo descritivo, nacional e institucional, transversal de caráter descritivo e analítico, aplicado à clínica, realizado na USF Alpha, pertencente ao Agrupamento de Centros de Saúde (ACeS) do Baixo Vouga. A população contemplada correspondeu a todos os utentes que recorreram à CA da USF Alpha no período de janeiro de 2014 a dezembro de 2017. A amostra estudada compreendeu todos os episódios em que um utente pertencente a essa população recorreu à CA da USF no período delineado. Não foram estabelecidos critérios de exclusão, mas em caso de omissão de dados ou erro detetado nos dados recolhidos, esses epi- sódios foram assumidos como inválidos para a análise da variável em questão, tendo sido excluídos.

Os dados relativos à CA foram recolhidos das plataformas informáticas Sclínico®e MIM@UF®. Para o estudo foram recolhidos os seguintes dados: idade e sexo dos utentes; distribuição temporal da CA ao longo do dia, da semana, mês e ano civil; frequência da CA por utente; identificação do médico e enfermeiro que observaram o utente; tipologia da consulta (CA realizada pelo médico de família ou SIS); motivo de consulta/ /diagnóstico observado na CA; encaminhamento dos utentes após a CA.

A análise dos dados obtidos foi feita através de tratamento estatístico apropriado, utilizando o programa informático Statistical Package for the Social Sciences (SPSS®), v. 25.0. Para a análise descritiva, frequências e percentagens foram usadas para caracterizar variáveis categóricas, tendo sido usado a mediana e amplitude interquartil para as variáveis contínuas com distribuição assimétrica e média e desvio-padrão para variáveis contínuas de distribuição normal. Além disso, foram também usadas formas de estatística inferencial, usando-se assim testes não paramétricos para comparar medianas de variáveis numéricas contínuas entre dois (teste de Mann-Whitney) ou mais (teste de Kruskal-Wallis) grupos e para comparar as frequências de variáveis entre dois grupos (teste de Qui-Quadrado), usando a correção de Bonferroni, quando aplicável. Foi admitida um nível de significância de 0,01.

Obteve-se parecer favorável da Comissão de Ética da Administração Regional de Saúde (ARS) do Centro, bem como a autorização do Diretor Executivo do ACeS Baixo Vouga. Foi criado um código aleatório para codificação dos utentes que recorreram à CA, de modo a garantir a anonimização, tendo-se assim garantido a confidencialidade e anonimização dos dados recolhidos.

\section{RESULTADOS}

A USF Alpha é composta por seis médicos, seis enfermeiros e cinco secretários clínicos, estando disponível diariamente ao utente CA das 8 horas da manhã às 20 horas da noite, tendo cada consulta a duração de 10 minutos. Da população inscrita na USF Alpha ( $n=10.442$ utentes, informação recolhida da plataforma informática MIM@UF® relativos a dezembro de 2017), de 1 de janeiro de 2014 a 31 de dezembro de 2017, 7.257 


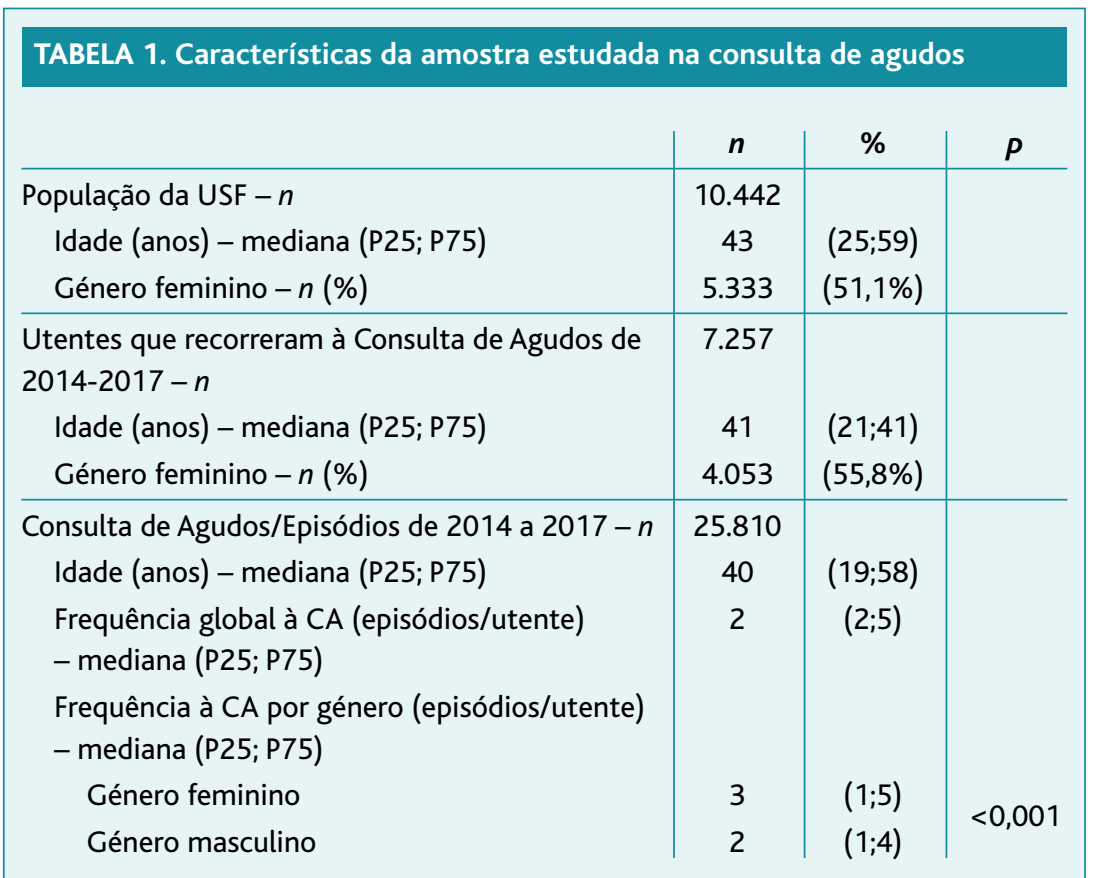

utentes recorreram à $\mathrm{CA}$, independentemente do número de vindas de cada utente à consulta durante esse período. Desses utentes 55,8\% ( $n=4.053)$ eram do género feminino, com uma mediana de idades de 41 anos (Tabela 1).

De 1 de janeiro de 2014 a 31 de dezembro de 2017 registaram-se 25.810 episódios de CA na USF Alpha, $60,4 \%$ desses episódios ( $n=15.596)$ foram de utentes do género feminino e $0,23 \%$ ( $n=59$ ) dos episódios foram excluídos do estudo por dados incompletos (Tabela 1). A mediana das idades dos utentes/episódio foi de 40 anos. As crianças com idades inferiores a 10 anos, que representam $8,2 \%$ da população inscrita na USF Alpha, foram responsáveis por $16,0 \%$ de todos os episódios de CA no período estudado. Por seu turno, o género feminino a partir da $3^{\text {a }}$ década de vida apresentou cerca do dobro da frequência em CA em relação aos homens das mesmas idades (Figura 1).

A frequência global à CA apresentou uma mediana de dois episódios/utente (P25; P75 2;5; mínimo 1, máximo 39), com uma frequência individual à CA estatisticamente diferente entre género masculino e feminino $(p<0,001)$, sendo superior no género feminino (três episódios/utente no género feminino $v s$ dois episódios/utente no género masculino) (Tabela 1). Ao longo dos anos de 2014 a 2017 a frequência dos episódios de CA manteve-se constante.

O primeiro trimestre do ano foi o que registou mais episódios ( $n=7.781$, $30,1 \%$ ), sendo janeiro o mês com maior frequência de CA $(n=2.823$, $11,3 \%)$. Julho, agosto e setembro apresentaram a menor frequência à $\mathrm{CA}$ (julho $n=1.857,7,2 \%$; agosto $n=1.891$, $7,3 \%$; setembro $n=1.952,7,6 \%$ ) (Figura 2). A segunda-feira foi o dia da semana em que os utentes recorreram mais à CA e a sexta-feira o dia em que recorreram menos, com frequências, respetivamente, de $27,2 \%(n=7.050) \mathrm{e}$ $15,8 \%(n=4.062)$. As oito horas da manhã foi o horário preferido dos utentes, tendo sido o período do dia que registou mais episódios de CA $(n=5.272,20,5 \%)$ e as 19 horas foi o horário com menor afluência $(n=1.005,3,9 \%)$ (Figuras 3A, B e C).

No seu total, as classificações por grupos da Classificação Internacional de Cuidados de Saúde Primários (ICPC-2) com maior frequência foram, por ordem decrescente, os problemas do aparelho respiratório ( $n=7.035,33,3 \%)$, os problemas do sistema músculo-esquelético ( $n=3.254,15,4 \%)$, os problemas do aparelho digestivo ( $n=2.531,15,4 \%$ ), os problemas da pele ( $n=2.090,9,9 \%)$ e os problemas do aparelho urinário ( $n=1.200,5,7 \%)$. Com base na codificação do ICPC-2, as dez patologias mais prevalentes em contexto de CA foram, por ordem decrescente, a infeção aguda do aparelho respiratório superior (R74) $(n=3.744,17,8 \%)$, amigdalite aguda (R76) ( $n=1.326,6,3 \%)$, cistite/infeção urinária (U71) ( $n=987,4,7 \%)$, gastroenterite, presumível infeção (D73) ( $n=846,4,0 \%)$, bronquite/bronquiolite aguda (R78) ( $n=737,3,5 \%)$, síndroma da coluna com irradiação de dor (L86) ( $n=559,2,7 \%)$, otite média aguda/miringite (H71) ( $n=462,2,2 \%)$, síndroma da coluna sem irradiação de dor (L84) ( $n=388,1,8 \%$ ), gripe (R80) ( $n=361,1,7 \%)$ e infeção da pele (S76) $(n=313,1,5 \%)$, totalizando $46,2 \%$ de toda a patologia observada na CA (Tabela 2). A patologia do foro respiratório foi a única que apresentou variação temporal considerável ao 


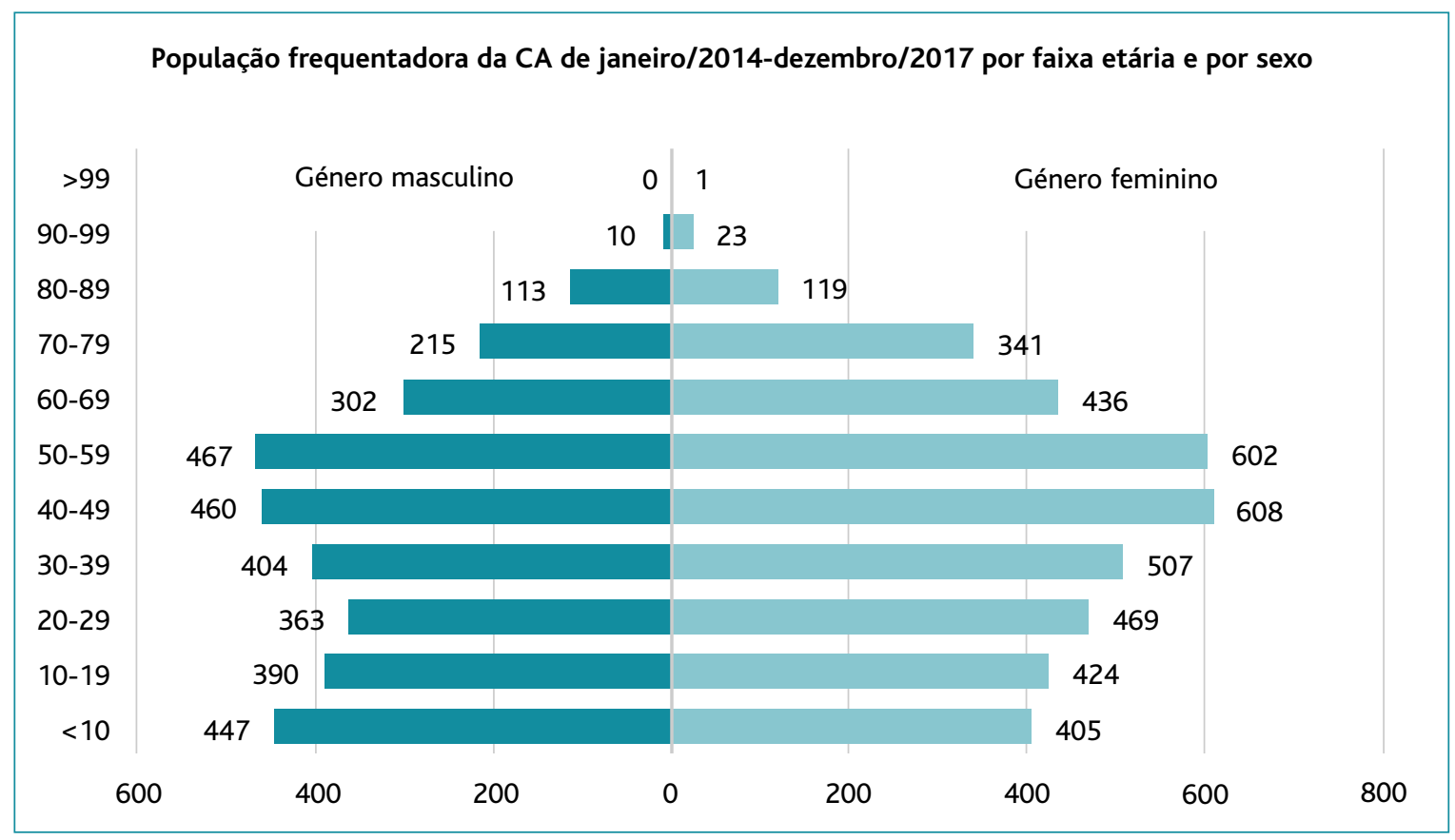

Figura 1. População frequentadora da CA de janeiro de 2014 a dezembro de 2017 por faixa etária e por sexo.

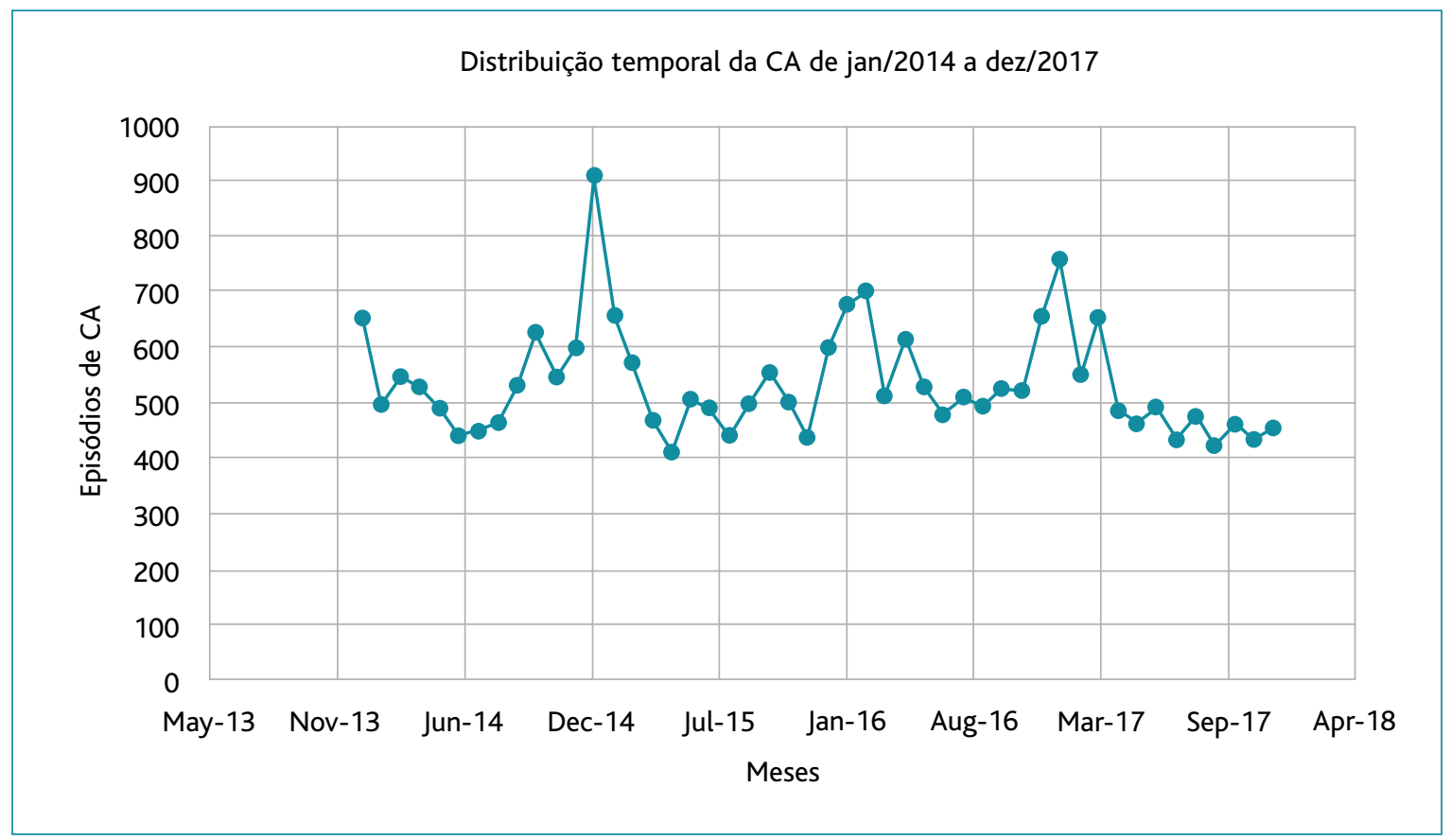

Figura 2. Distribuição temporal dos episódios de consulta de agudos de janeiro de 2014 a dezembro de 20217.

longo dos meses do ano, verificando-se que foi a patologia mais prevalente ao longo dos meses de Outono-Inverno (Figura 4).
Há diferenças estatisticamente significativas quando analisadas as patologias por grupos de patologia do ICPC-2 de acordo com o género e idade $(p<0,001$, 

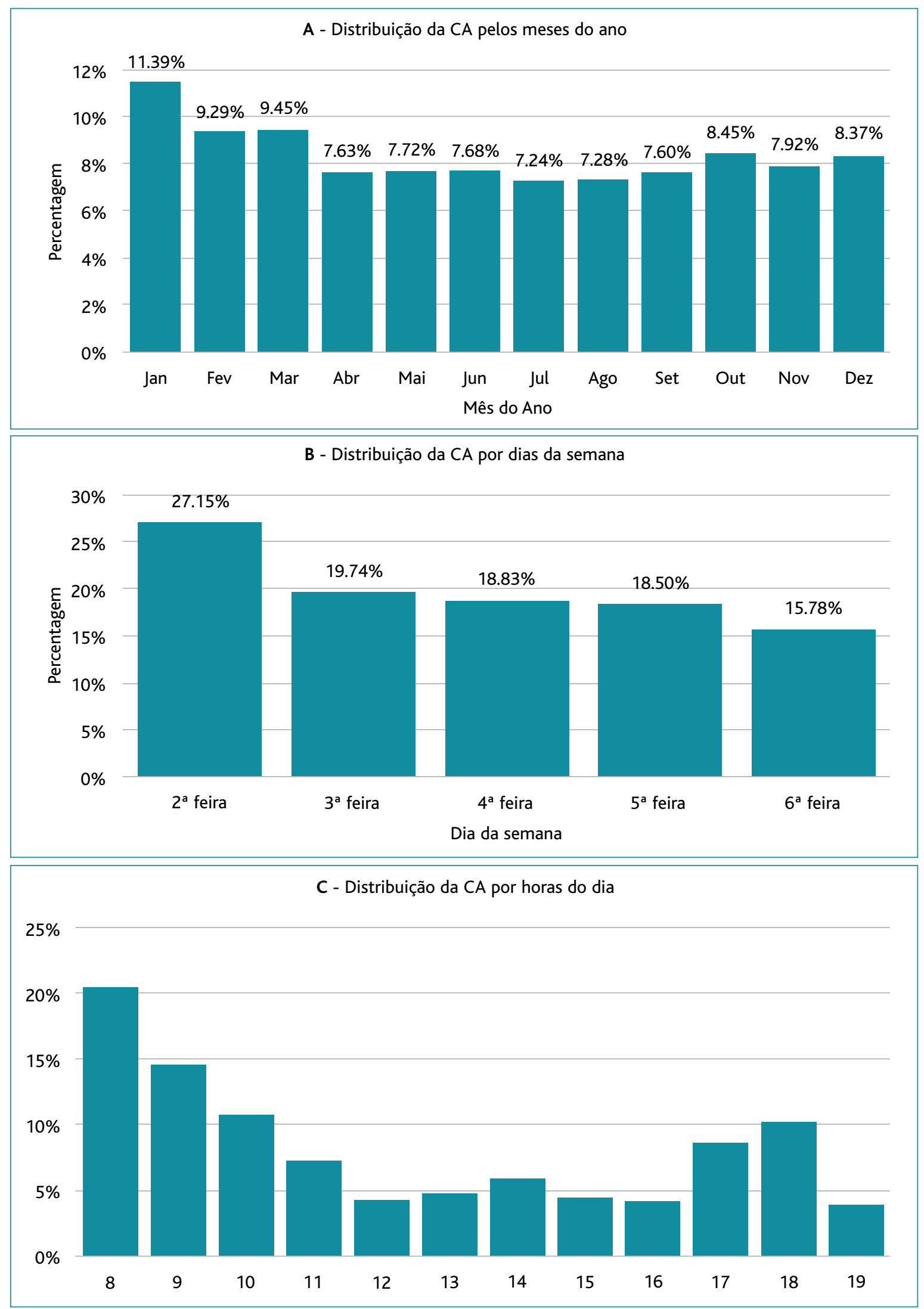

Figura 3. A, B, C. Distribuição da CA da USF por meses do ano (A), dias da semana (B) e horas do dia (C). 
TABELA 2. Frequência absoluta e relativa, por sexo, dos grupos de patologias e de acordo com a codificação do ICPC-2 na consulta de adultos

\begin{tabular}{|c|c|c|c|c|c|c|c|c|}
\hline & & \multicolumn{2}{|c|}{ Feminino } & \multicolumn{2}{|c|}{ Masculino } & \multirow[b]{2}{*}{$p$} & \multicolumn{2}{|c|}{ Total } \\
\hline & & $n$ & $\%$ & $n$ & $\%$ & & $n$ & $\%$ \\
\hline \multirow{18}{*}{$\begin{array}{l}\text { Diagnóstico } \\
\text { ICPC-2 }\end{array}$} & Geral e inespecífico & 522 & $4,1 \%$ & 414 & $5,0 \%$ & 0,001 & 936 & $4,4 \%$ \\
\hline & $\begin{array}{l}\text { Sangue, Sistema hematopoiético, } \\
\text { Linfático, Baço }\end{array}$ & 78 & $0,6 \%$ & 28 & $0,3 \%$ & 0,007 & 106 & $0,5 \%$ \\
\hline & Aparelho digestivo & 1.454 & $11,3 \%$ & 1.077 & $13,0 \%$ & $<0,001$ & 2.531 & $12,0 \%$ \\
\hline & Olho & 275 & $2,1 \%$ & 213 & $2,6 \%$ & 0,045 & 488 & $2,3 \%$ \\
\hline & Ouvido & 532 & $4,1 \%$ & 373 & $4,5 \%$ & & 905 & $4,3 \%$ \\
\hline & Aparelho circulatório & 375 & $2,9 \%$ & 175 & $2,1 \%$ & 0,001 & 550 & $2,6 \%$ \\
\hline & Sistema músculo-esquelético & 1.929 & $15,0 \%$ & 1.325 & $16,0 \%$ & 0,067 & 3.254 & $15,4 \%$ \\
\hline & Sistema neurológico & 305 & $2,4 \%$ & 139 & $1,7 \%$ & 0,001 & 444 & $2,1 \%$ \\
\hline & Psicológico & 561 & $4,4 \%$ & 241 & $2,9 \%$ & $<0,001$ & 802 & $3,8 \%$ \\
\hline & Aparelho respiratório & 4.014 & $31,3 \%$ & 3.021 & $36,4 \%$ & $<0,001$ & 7.035 & $33,3 \%$ \\
\hline & Pele & 1.269 & $9,9 \%$ & 821 & $9,9 \%$ & 0,995 & 2.090 & $9,9 \%$ \\
\hline & Endócrino, metabólico e nutricional & 47 & $0,4 \%$ & 79 & $1,0 \%$ & $<0,001$ & 126 & $0,6 \%$ \\
\hline & Aparelho urinário & 966 & $7,5 \%$ & 234 & $2,8 \%$ & $<0,001$ & 1.200 & $5,7 \%$ \\
\hline & $\begin{array}{l}\text { Gravidez, Parto, Planeamento } \\
\text { familiar }\end{array}$ & 80 & $0,6 \%$ & & & & 80 & $0,4 \%$ \\
\hline & Aparelho genital feminino & 383 & $3,0 \%$ & & & & 385 & $1,8 \%$ \\
\hline & Aparelho genital masculino & & & 126 & $1,5 \%$ & & 127 & $0,6 \%$ \\
\hline & Problemas sociais & 43 & $0,3 \%$ & 33 & $0,4 \%$ & 0,459 & 76 & $0,4 \%$ \\
\hline & Total & 12.834 & $100,0 \%$ & 8.301 & $100,0 \%$ & & 21.135 & $100,0 \%$ \\
\hline \multirow{12}{*}{$\begin{array}{l}\text { Top } 10 \text { de } \\
\text { diagnósticos }\end{array}$} & R74 - Infeção aguda do aparelho & & & & & & & \\
\hline & respiratório superior & 2.062 & $16,1 \%$ & 1.682 & $20,3 \%$ & & 3.744 & $17,8 \%$ \\
\hline & R76 - Amigdalite aguda & 796 & $6,2 \%$ & 529 & $6,4 \%$ & & 1326 & $6,3 \%$ \\
\hline & U71 - Cistite/infeção urinária & 852 & $6,7 \%$ & 135 & $1,6 \%$ & & 987 & $4,7 \%$ \\
\hline & $\begin{array}{l}\text { D73 - Gastroenterite, presumível } \\
\text { infeção }\end{array}$ & 445 & $3,5 \%$ & 401 & $4,8 \%$ & & 846 & $4,0 \%$ \\
\hline & R78 - Bronquite/Bronquiolite aguda & 447 & $3,5 \%$ & 290 & $3,5 \%$ & & 737 & $3,5 \%$ \\
\hline & $\begin{array}{l}\text { L86 - Síndroma da coluna com } \\
\text { irradiação de dor }\end{array}$ & 219 & $2,5 \%$ & 244 & $2,9 \%$ & & 559 & $2,7 \%$ \\
\hline & H71 - Otite média aguda/Miringite & 253 & $2,0 \%$ & 209 & $2,5 \%$ & & 462 & $2,2 \%$ \\
\hline & $\begin{array}{l}\text { L84 - Síndroma da coluna sem } \\
\text { irradiação de dor }\end{array}$ & 219 & $1,7 \%$ & 169 & $2,0 \%$ & & 388 & $1,8 \%$ \\
\hline & R80 - Gripe & 182 & $1,4 \%$ & 179 & $2,2 \%$ & & 361 & $1,7 \%$ \\
\hline & S76 - Infeção da pele & 176 & $1,5 \%$ & 118 & $1,4 \%$ & & 313 & $1,5 \%$ \\
\hline & Total do Top 10 face ao total de CA & 5.651 & $45,1 \%$ & 3.956 & $62 \%$ & & 9723 & $46,2 \%$ \\
\hline
\end{tabular}




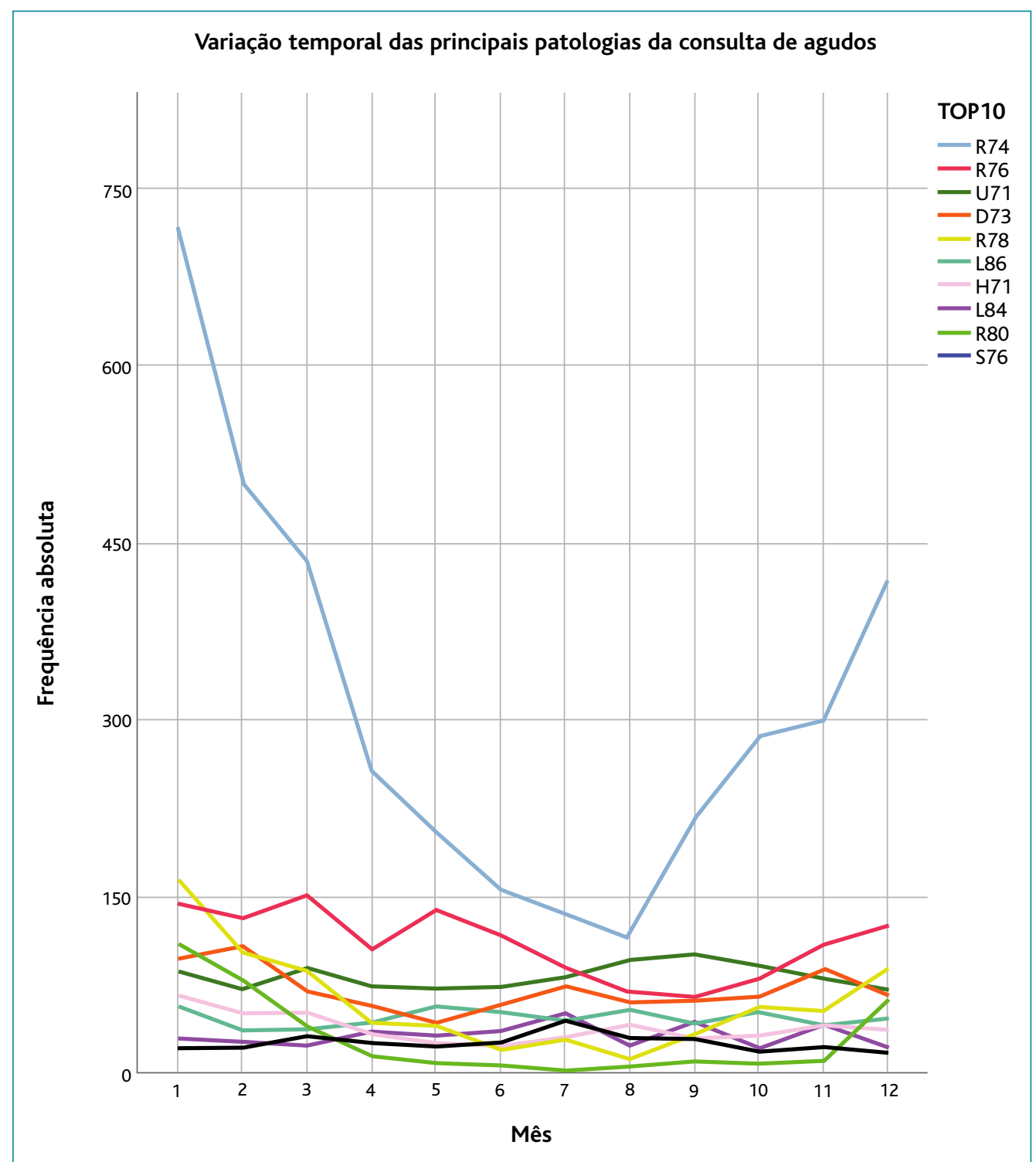

Figura 4. Variação temporal ao longo do ano da frequência absoluta das 10 patologias mais frequentes na consulta de agudos.

Legenda: R74 - Infeção aguda do aparelho respiratório superior; R76 - Amigdalite aguda; U71 - Cistite/infeção urinária; D73 - Gastroenterite, presumível infeção; R78 - Bronquite/bronquiolite aguda; L86 - Síndroma da coluna com irradiação de dor; H71 - Otite média aguda/miringite; L84 - Síndroma da coluna sem irradiação de dor; R80 - Gripe; S76 - Infeção da pele.

respetivamente). Assim, o género masculino apresentou mais frequentemente, e de modo significativo, problemas gerais e inespecíficos $(p=0,001)$, problemas do aparelho digestivo $(p<0,001)$, do aparelho respiratório $(p<0,001)$, do sistema endócrino, metabólico e nutricional $(p<0,001)$. Por seu lado, o género feminino apresentou mais significativamente problemas do sangue, sistema hematopoiético, linfático, baço ( $p=0,007)$, aparelho circulatório $(p<0,001)$, sistema neurológico $(p=0,001)$, psicológico $(p<0,001)$ e aparelho urinário $(p<0,001)$ (Tabela 2). Em concreto, destaca-se que a cistite/infeção urinária foi mais comum no género feminino por oposição ao género masculino $(6,7 \% v s$ $1,6 \%$ ), assim como os problemas do foro psicológico, 


\begin{tabular}{|c|c|c|c|c|c|c|c|c|}
\hline & & \multicolumn{2}{|c|}{ CA - MF } & \multicolumn{2}{|c|}{ CA - SIS } & \multirow[t]{2}{*}{$p$} & \multicolumn{2}{|c|}{ Total } \\
\hline & & $n$ & $\%$ & $n$ & $\%$ & & $n$ & $\%$ \\
\hline \multirow[t]{7}{*}{ Destino: } & Domicílio & 8.380 & $92,8 \%$ & 9.275 & $92,0 \%$ & 0,026 & 17.655 & $92,39 \%$ \\
\hline & Urgência & 405 & $4,5 \%$ & 622 & $6,2 \%$ & 0,001 & 1.027 & $5,38 \%$ \\
\hline & Tratamento de enfermagem & 101 & $1,1 \%$ & 82 & $0,8 \%$ & 0,030 & 183 & $0,96 \%$ \\
\hline & Não responde a chamada & 58 & $0,6 \%$ & 56 & $0,6 \%$ & 0,522 & 114 & $0,60 \%$ \\
\hline & Consulta hospitalar não urgente & 68 & $0,8 \%$ & 37 & $0,4 \%$ & $<0,001$ & 105 & $0,55 \%$ \\
\hline & INEM & 14 & $0,2 \%$ & 11 & $0,1 \%$ & 0,417 & 25 & $0,13 \%$ \\
\hline & Total & 9.026 & $100,0 \%$ & 10.083 & $100,0 \%$ & & 19.109 & $100 \%$ \\
\hline
\end{tabular}

Legenda: CA-MF = Consulta de agudos realizada pelo médico de família do utente; CA-SIS = Consulta de agudos realizada em sistema de intersubstituição.

nomeadamente a perturbação depressiva $(1,9 \%$ s $0,6 \%)$.

Quando analisadas as patologias mais frequentes da CA por idades segundo a divisão das unidades ponderadas nos CSP, pode-se constatar que, à semelhança do que sucede na frequência por sexo, as patologias mais frequentes mantiveram-se grosseiramente as mesmas para os mesmos escalões etários. A referir, a otite média aguda/miringite e a gastroenterite foram mais comuns até aos seis anos, diminuindo de frequência com a idade. Por outro lado, a cistite/infeção urinária e os problemas do sistema músculo-esquelético foram menos comuns até aos seis anos, tendo apresentado maior frequência a partir dessa idade.

Relativamente à orientação após a CA, dentro das patologias mais frequentemente referenciadas ao serviço de urgência (SU), considerando a sua frequência relativa de diagnóstico na $\mathrm{CA}$, pode-se constatar que os sinais/sintomas ou patologias com maior taxa de referenciação para o SU foram: dificuldade respiratória/dispneia (R02) ( $n=10,83,3 \%)$, apendicite (D88) ( $n=16$, 80,0\%), sensação de pressão/aperto atribuído ao coração (K02) ( $n=12,75,0 \%)$, pielonefrite/pielite (U70) ( $n=22,57,9 \%$ ), doença ou condição de natureza ou localização não especificadas (A99) ( $n=52,50,0 \%)$, insuficiência cardíaca (K77) ( $n=13,43,3 \%)$, dor abdominal localizada (D06) ( $n=61,42,7 \%)$, desmaio/síncope (A06) $(n=10,40,0 \%)$, pneumonia (R81) $(n=15,39,5 \%)$ e flebite e tromboflebite (K94) $(n=25,30,9 \%)$. De notar que ou- tras patologias foram referenciadas com maior frequência absoluta ao SU, mas com baixa frequência relativa, como por exemplo a bronquite/bronquiolite aguda (R78) ( $n=61,9,4 \%)$ ou o traumatismo do aparelho músculo-esquelético não especificado (L81) ( $n=44$, $23,4 \%$ ). Assim sendo, por exemplo, ainda que se observem muitos utentes com bronquite/bronquiolite aguda, esta não correspondeu à patologia que, quando observada em CSP, foi mais frequentemente encaminhada para o SU (Tabela 3).

Analisando de igual modo, os problemas com maior taxa de referenciação para o INEM foram: sinal/sintoma do sistema nervoso (N29) $(n=1,50,0 \%)$, enfarte agudo do miocárdio (K75) ( $n=1,50,0 \%)$, isquemia cerebral transitória (K89) ( $n=1,33,3 \%$ ), sensação de pressão/ /aperto atribuído ao coração (K02) ( $n=3,18,8 \%)$ e palpitações/perceção do batimento cardíaco (K04) ( $n=3$, 11,5\%) (Tabela 3).

Pela variada dispersão de problemas com maior referenciação para consulta hospitalar não urgente apresentam-se os problemas mais referenciados atendendo à sua frequência absoluta de referenciação, a notar: neoplasia maligna do cólon/reto (D75) ( $n=6,66,7 \%)$, neoplasia cutânea benigna/incerta (S79) $(n=4,50,0 \%)$, nevo/sinal da pele (S82) $(n=4,20,0 \%)$, cerúmen no ouvido em excesso (H81) ( $n=10,12,5 \%)$ e quisto pilonidal/fístula (S85) ( $n=4,9,8 \%)$ (Tabela 3).

Quando comparadas as proporções dos destinos dos utentes entre médicos verificou-se que há diferenças 
estatisticamente significativas quando se compara o destino atribuído pelos médicos $(p<0,001)$, havendo diferenças de atuação entre o médico de família do utente em CA e o médico da USF que consulta esporadicamente o utente em SIS. Quando analisado cada destino em particular verifica-se que o médico de família, em contexto de CA, encaminhou mais o seu utente para o domicílio $(92,8 \%$ vs $92 \%, p=0,026)$, para o tratamento de enfermagem $(1,1 \% v s 0,8 \%, p=0,030)$ e para consulta hospitalar não urgente $(0,8 \%$ vs $0,4 \%, p<0,001)$. De modo oposto, o médico de família, face a outro médico da USF que observa o utente em SIS, referenciou menos para o SU $(4,5 \%$ vs $6,2 \%, p<0,001)$, não havendo diferença estatisticamente significativa entre médicos quando analisada a necessidade chamar o INEM $(0,2 \%$ vs $0,1 \%, p=0,417$ ) (Tabela 3).

\section{DISCUSSÃO}

Trata-se de um estudo inovador atendendo à considerável dimensão amostral e ao tempo de estudo, no decorrer de quatro anos, o que permite colmatar eventuais variações na frequência à CA entre os diferentes anos, nomeadamente ausências prolongadas de profissionais da USF ou pontuais surtos epidémicos. Por este motivo, não há estudos nacionais que façam uma análise tão detalhada e que permita uma caracterização de um modelo de CA. Ainda assim, verifica-se que a caracterização do perfil do utilizador da CA da USF se enquadra em investigações científicas nacionais sobre a mesma temática. ${ }^{5-7}$

Este estudo, atendendo à sua caracterização de acordo com a distribuição temporal da CA por anos, trimestres, meses, dias da semana e horas, permite adequar a oferta da CA de uma USF às suas necessidades, de modo a garantir ao máximo uma boa acessibilidade. Esta avaliação das necessidades da USF acaba por permitir adequar a oferta da USF às necessidades da comunidade em questão, além de melhorar a satisfação do utente e reduzir o recurso indevido a CA.

Por outro lado, o estudo apresenta algumas limitações relacionadas com o facto de a classificação do ICPC-2 ser algo limitada na codificação exata de alguns motivos de consulta/patologias, o que cria alguns constrangimentos na perceção exata da patologia que realmente motivou a observação em alguns episódios agudos. Além disso, a caracterização sociodemográfica do utente utilizador de CA poderia ter sido mais abrangente, nomeadamente no que diz respeito à sua escolaridade, situação profissional, agregado familiar e presença de multipatologia.

$\mathrm{Na}$ análise detalhada dos dados destaca-se a diferença no número de episódios de CA de acordo com o género, o que remete para a grande necessidade de melhorar e individualizar, de acordo com o género, as estratégias de educação para a saúde, nomeadamente no que diz respeito à patologia aguda. Além disso, verifica-se que as principais patologias observadas em contexto de CA na USF dizem respeito à patologia infeciosa/aguda, o que está de acordo com o que se pretende numa consulta desta tipologia, sendo crucial a correta difusão, junto dos profissionais de saúde e dos utentes, de boas práticas de saúde e higiene para gestão destas patologias, orientação terapêutica, com especial atenção à prescrição antibiótica.

À semelhança do verificado em estudos de investigação da mesma temática, ${ }^{6-7}$ a patologia mais prevalente foi a infeciosa do foro respiratório, em particular infeções das vias aéreas superiores, as quais na maioria dos casos se deve a agentes víricos, pelo que o seu tratamento, pelo menos nas primeiras 48-72h, é essencialmente sintomático. Deste modo, deve-se capacitar o utente, tanto quanto possível, na autogestão da sua patologia de modo a melhorar a acessibilidade e por forma a garantir uma cooperação entre médico e utente, não esquecendo a importância de informar para os verdadeiros sinais de alarme que devem, de acordo com as diferentes patologias, motivar o utente a ser observado nos cuidados de saúde.

Por outro lado, a patologia osteodegenerativa foi uma das dez patologias mais comummente observadas em CA. Atendendo a que frequentemente se tratam de problemas já conhecidos e crónicos, como as síndromas da coluna com ou sem irradiação da dor, realça-se a importância do adequado controlo da doença, em particular da dor e devida orientação da mesma em caso de agudização, bem como da educação dos doentes com estas patologias para melhor perceber como gerir a sua doença e quando recorrer aos serviços de saúde.

Os principais sinais/sintomas ou patologias referenciadas para o SU são situações que exigem estudo com meios auxiliares de diagnóstico de modo urgente 
para estudo ou que necessitam de tratamento médico urgente para tratamento e estabilização do quadro clínico. Para além disso, os principais motivos de chamada do INEM foram causas do foro cardíaco, que se prendem muitas vezes com diagnóstico diferencial com enfarte agudo do miocárdio, portanto, uma situação urgente que exige exclusão rápida e eventual tratamento médico hospitalar urgente.

Por outro lado, os dados reforçam as discrepâncias de atuação entre médicos. Este resultado denota que a prática clínica varia de médico para médico e incita à estandardização de procedimentos em contexto de CA e, em particular, de patologia aguda, para consolidar também a educação da população no que diz respeito ao objetivo da CA. Muito deste processo passa pelo estabelecimento de critérios mais definidos para a referenciação para o SU. O facto de o médico de família orientar menos para o serviço de urgência, mas mais para o domicílio, tratamento de enfermagem e consulta hospitalar não urgente, denota o papel mais próximo do próprio médico de família face aos outros médicos de uma mesma USF. Assim sendo, o médico de família possui já informação prévia que permite a integração do conhecimento do utente na sua vertente biopsicossocial, permitindo também uma maior coordenação com os serviços de apoio, neste caso, com enfermagem e na interligação com consulta hospitalar. $\mathrm{O}$ facto de não se detetarem diferenças estatisticamente significativas na orientação para o INEM reforça a igualdade na identificação dos casos potencialmente graves, com necessidade de abordagem urgente/emergente a nível de cuidados hospitalares.

Constata-se que a própria patologia aguda tem diferentes níveis de gravidade e, perante isso, pode implicar apenas gestão da doença pelo próprio doente, observação em contexto de CA nos CSP ou pode mesmo implicar observação em cuidados de saúde secundários (CSS). Deste modo, o presente estudo torna evidente a necessidade de haver algoritmos de decisão e de estratificação de gravidade das situações agudas que motivam realmente o recurso a CA ao nível dos CSP ou a necessidade de recurso a CSS.
Além disso, pelo facto de se tratar de um estudo com uma considerável dimensão amostral, abre a possibilidade de estudo da CA de um modo mais abrangente, por forma a que o tema CA seja mais amplamente estudado com um esqueleto geral replicável em todas as USF e com adequação à população que cada USF serve.

\section{REFERÊNCIAS BIBLIOGRÁFICAS}

1. Pisco L.A reforma dos cuidados de saúde primários [Internet]. Cad Economia. 2007;(80):60-6.Available from: http://www2.portaldasaude.pt/ NR/rdonlyres/9A05F533-B7AE-4256-9F80-7FDD3E7C4FC7/0/CE80 Luis_Pisco.pdf

2. Allen J, Gay B, Crebolder H, Heyrman J, Svab I, Ram P, et al. A definição Europeia de medicina geral e familiar (clínica geral/medicina familiar) [The European definition of family medicine (general practice/family medicine). Rev Port Clin Geral. 2005;21(5):511-6. Portuguese

3. Missão para os Cuidados de Saúde Primários. Consulta aberta:'consulta de agudos, não programada' versus consulta aberta no hospital e centro de saúde [Internet]. Lisboa: MCSP; 2007. Available from: https://www2.acss.min-saude.pt/Portals/O/Consulta_Aberta_MCSP_ 20071213.pdf

4. Despacho Normativo n. ${ }^{\circ}$ 9/2006, de 16 de fevereiro. Diário da República. I Série-B(34).

5. Carraça I, Gala AB, Gago HS, Gameiro L. Atendimento complementar e acessibilidade [After hours care and accessibility]. Rev Port Clin Geral. 2002;18(5):271-80. Portuguese

6. Palma J. Problemas de saúde urgentes: a quem recorrem os utentes e o que influencia a sua escolha [Urgent health problems: the services patients choose and what influences their choices]. Rev Port Clin Geral. 2002;18(5):283-97. Portuguese

7. Ramos AA, Proença I, Magalhães J, Grijó L, Beirão L, Peixoto MJ, et al. Caracterização das consultas abertas [Walk-in appointments: a crosssectional descriptive analysis in a Portuguese family health unit]. Rev Port Med Geral Fam. 2018;34(6):361-9. Portuguese

\section{CONFLITO DE INTERESSES}

Os autores declaram não possuir quaisquer conflitos de interesse.

\section{ENDEREÇO PARA CORRESPONDÊNCIA}

Ana Aires

E-mail: anaaires1992@gmail.com

https://orcid.org/0000-0002-1288-6609

Recebido em 23-02-2020

Aceite para publicação em 21-05-2020 


\section{ABSTRACT}

\section{ACUTE DISEASE IN PRIMARY HEALTH CARE: A CROSS-SECTIONAL DESCRIPTIVE ANALYSIS IN A PORTUGUESE FAMILY HEALTH UNIT}

Objective: To characterize same-day appointments (SDA) carried out in a Family Health Unit (FHU) over four years, according to the frequency of appointments/diagnosis and the user's destination.

Study type: Cross-sectional, descriptive, and analytical study, applied to the clinic.

Location: FHU Alpha, ACeS Baixo Vouga.

Population: Patients who came to SDA at USF from January-2014 to December-2017.

Methods: All episodes of SDA over four years $(n=25,810)$ were studied, namely sex, age, the temporal distribution of SDA; frequency of SDA; diagnosis, and destination of patients. A descriptive and inferential analysis was performed with non-parametric tests using the software Statistical Package for the Social Sciences (SPSS $\left.{ }^{\circledR}\right)$, v. 25.0.

Results: Of the episodes of SDA, the majority were women $(n=15,596,60.4 \%, p<0.001)$, with a median age of 40 years and frequency/person of two episodes in the period described. Most SDAs took place in the first quarter of the year, in January, on Monday, and in the early hours of the day. The most prevalent pathologies in the context of SDA were acute infection of the upper respiratory system $(n=3,744,17.8 \%)$, acute tonsillitis $(n=1,326,6.3 \%)$, cystitis/urinary infection $(n=987,4.7 \%)$, gastroenteritis ( $n=846,4.0 \%$ ) and acute bronchitis/bronchiolitis $(n=737,3.5 \%)$. There were significant differences in the referral between the patient's family doctor and care by another doctor, referring the latter more to the emergency department $(p<0.001)$ and there were no differences regarding the need to call emergency $(p=0.417)$.

Conclusion: There were differences in health care between family doctors, creating the need for decision algorithms and severity stratification as a strategy to improve accessibility and quality of care provided in primary health care. Health education for patients is a fundamental strategy to work, mostly in the context of acute pathology.

Keywords: Primary health care; Acute disease; Walk-in appointments; Portugal. 